\title{
Oriented Matroids for Shape Representation and Indexing
}

\author{
E. Staffetti ${ }^{1}$, A. Grau ${ }^{2}$, F. Serratosa ${ }^{3}$, and A. Sanfeliu ${ }^{1}$ \\ 1 Institut de Robòtica i Informàtica Industrial (CSIC-UPC) \\ Llorens i Artigas 4-6, 08028 Barcelona Spain \\ \{estaffetti, asanfeliu\}@iri.upc.es \\ 2 Departament d'Enginyeria de Sistemes Automàtica i Informàtica Industrial (UPC) \\ Pau Gargallo 5, 08028 Barcelona Spain \\ antoni.grau@upc.es \\ 3 Departament d' Enginyeria Informàtica i Matemàtiques (URV) \\ Av. Paisos Catalanes, 2643007 Tarragona Spain \\ Francesc.Serratosa@etse.urv.es
}

\begin{abstract}
In this paper a novel method for indexing views of $3 \mathrm{D}$ objects is presented. The topological properties of the regions of the segmented images of the objects are used to define an index based on oriented matroid theory. Oriented matroids, which are projective invariants, encode incidence relations and relative position of the elements of the image and give local and global topological information about their spatial distribution. This indexing technique is applied to 3D object hypothesis generation from single views to reduce the number of candidates in object recognition processes.
\end{abstract}

\section{Introduction}

In this paper a new method for indexing views of $3 \mathrm{D}$ objects is presented. It is applied to 3D object hypothesis generation to reduce the number of candidates in object recognition processes. Given a set of views of different objects, the problem of object recognition using a single image can be regarded as the problem of finding a subset of the set of regions in the image with a relational structure identical to that of a member of the set of views. The standard way to reduce the complexity of model matching is subdividing the problem into a hypothesis generation followed by a verification. To be of interest in object recognition the hypothesis generation should be relatively fast although imprecise in which several possible candidates for matching are generated. In this way the verification can be carried out using a more complex and, therefore, slower procedure [1] over a reduced number of candidates. The hypothesis generation can be made very efficient if it is formulated as an indexing problem where views of a set of $3 \mathrm{D}$ objects are stored into a table that is indexed by some function of the views themselves.

In this paper an indexing technique based on oriented matroid theory is presented. More precisely, the topological properties of the regions of the segmented 
views of $3 \mathrm{D}$ objects are encoded into a data structure called set of cocircuits. The sets of cocircuits of the different views of a database merged together are used as an index of the database itself. The set of cocircuits, that are one of the several combinatorial data structures called oriented matroids, encode incidence relations and relative position of the elements of the image and give local and global topological information about their spatial distribution. Since index tables are by definition discrete, the discrete nature of the combinatorial structure of the set of cocircuits nicely fits with this technique. This method is employed to the hypothesis generation for $3 \mathrm{D}$ object recognition from single views. The principal aspects of oriented matroid theory together with some applications were compiled in 1993 in the comprehensive monograph [2]. For shorter introductions see [3] or [4]. For another approach to shape representation and indexing based on combinatorial geometry see [5].

The paper is organized as follows: in Section 2 oriented matroids are introduced and their invariance properties are illustrated. In Sections 3 the proposed indexing method is described together with the strategy used for hypothesis generation. In Section 4 some experimental results obtained applying the proposed method to 3D object recognition are reported. Finally, Section 5 contains the conclusions.

\section{Oriented Matroids}

Oriented matroid theory is a broad setting in which the combinatorial properties of geometrical configurations can be described and analyzed. It provides a common generalization of a large number of different mathematical objects usually treated at the level of usual coordinates. In this section oriented matroids will be introduced over arrangements of points using two combinatorial data structures called chirotope and set of cocircuits.

\subsection{Oriented Matroids of Arrangements of Points}

Given a point configuration in $\mathbb{R}^{d-1}$ whose elements are the columns of the matrix $\mathbf{P}=\left(p_{1}, p_{2}, \ldots, p_{n}\right)$, the associated vector configuration is a finite spanning sequence of vectors $\left\{x_{1}, x_{2}, \ldots, x_{n}\right\}$ in $\mathbb{R}^{d}$ represented as columns of the matrix $X=\left(x_{1}, x_{2}, \ldots, x_{n}\right)$ where each point $p_{i}$ is represented in homogeneous coordinates as $x_{i}=\left(\begin{array}{c}p_{i} \\ 1\end{array}\right)$. To encode the combinatorial properties of the point configuration we can use a data structure called chirotope [4] which can be computed using the associated vector configuration $X$. The chirotope of $X$ is the map

$$
\begin{aligned}
\chi_{X}:\{1,2, \ldots, n\}^{d} & \rightarrow\{+, 0,-\} \\
\left(\lambda_{1}, \lambda_{2}, \ldots, \lambda_{d}\right) & \mapsto \operatorname{sign}\left(\left[x_{\lambda_{1}}, x_{\lambda_{2}}, \ldots, x_{\lambda_{d}}\right]\right)
\end{aligned}
$$

that assigns to each $d$-tuple of the vectors of the finite configuration $X$ a sign + or - depending on whether it forms a basis of $\mathbb{R}^{d}$ having positive or negative 


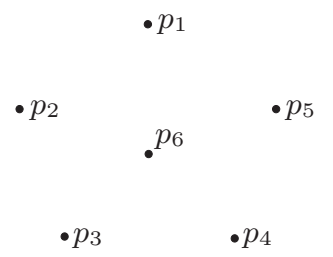

Fig. 1. A planar point configuration

Table 1. Vector configuration that corresponds to the planar point configuration represented in Fig. 1

\begin{tabular}{|c|c|c|}
\hline$x_{1}=(0,3,1)$ & $x_{2}=(-3,1,1)$ & $x_{3}=(-2,-2,1)$ \\
\hline$x_{4}=(2,-2,1)$ & $x_{5}=(3,1,1)$ & $x_{6}=(0,0,1)$ \\
\hline
\end{tabular}

orientation, respectively. This function assigns the value 0 to those $d$-tuples that do not constitute a basis of $\mathbb{R}^{d}$. The chirotope describes the incidence structure and relative position of the points of the arrangement with respect to the hyperplanes passing through them.

Example 1. Consider the point configuration represented in Fig. 1 whose associated vector configuration $X$ is given in Table 1 .

The chirotope $\chi_{X}$ of this vector configuration is given by the orientations listed in Table 2.

The element $\chi(1,2,3)=+$, for instance, indicates that, in the triangle formed by $p_{1}, p_{2}$, and $p_{3}$, these points are counterclockwise ordered. These orientations can be rearranged in an equivalent data structure called set of cocircuits of $X$ shown in Table 3. In this case, the set of cocircuits of $X$ is the set of all partitions generated by lines passing through two points of the configuration. For example, $(0,0,+,+,+,+)$ means that the points $p_{3}, p_{4}, p_{5}$, and $p_{6}$ lie on the same half plane determined by the line through the points $p_{1}$ and $p_{2}$. Changing the signs of the set of cocircuits we obtain an equivalent description of the arrangement of points.

Besides chirotopes and sets of cocircuits there are several data structures capable of encoding the combinatorial properties of a point configuration [4]. It

Table 2. Chirotope of the planar point configuration represented in Fig. 1

\begin{tabular}{|l|l|l|l|l|}
\hline$\chi(1,2,3)=+$ & $\chi(1,2,4)=+$ & $\chi(1,2,5)=+$ & $\chi(1,2,6)=+$ & $\chi(1,3,4)=+$ \\
\hline$\chi(1,3,5)=+$ & $\chi(1,3,6)=+$ & $\chi(1,4,5)=+$ & $\chi(1,4,6)=-$ & $\chi(1,5,6)=-$ \\
\hline$\chi(2,3,4)=+$ & $\chi(2,3,5)=+$ & $\chi(2,3,6)=+$ & $\chi(2,4,5)=+$ & $\chi(2,4,6)=+$ \\
\hline$\chi(2,5,6)=-$ & $\chi(3,4,5)=+$ & $\chi(3,4,6)=+$ & $\chi(3,5,6)=+$ & $\chi(4,5,6)=+$ \\
\hline
\end{tabular}


Table 3. Set of cocircuits of the planar point configuration represented in Fig. 1

\begin{tabular}{|c|c|c|}
\hline$(0,0,+,+,+,+)$ & $(0,-, 0,+,+,+)$ & $(0,-,-, 0,+,-)$ \\
\hline$(0,-,-,-, 0,-)$ & $(0,-,-,+,+, 0)$ & $(+, 0,0,+,+,+)$ \\
\hline$(+, 0,-, 0,+,+)$ & $(+, 0,-,-, 0,-)$ & $(+, 0,-,-,+, 0)$ \\
\hline$(+,+, 0,0,+,+)$ & $(+,+, 0,-, 0,+)$ & $(+,+, 0,-,-, 0)$ \\
\hline$(+,+,+, 0,0,+)$ & $(-,+,+, 0,-, 0)$ & $(-,-,+,+, 0,0)$ \\
\hline
\end{tabular}

can be proven that all of them are equivalent and are referred to as oriented matroids.

In the next section a method to represent with an oriented matroid the combinatorial structure of views of three dimensional objects will be presented. It will be used for indexing the image database in which they are stored.

\subsection{Oriented Matroid of Arrangements of Regions}

Extracting the oriented matroid of a view is not straightforward since the regions that form an image cannot be reduced to points, taking for example their centroids, without losing essential topological information for object recognition. Therefore, in the method presented in this paper the convex hull [6] of each region is used to represent the region itself. Then, pairs of non-overlapped convex regions resulting from this process are considered and their convex hulls are merged. The oriented matroid is extracted based on the spatial location of the other convex regions of the image with respect to the two lines arising when merging the convex hulls of two of them. Consider, for instance, the ordered pair of convex regions $(S, T)$ of the view $v_{1,1}$ of Fig. 3 . It is easy to see that the convex hull of these two convex planar non-overlapped polygons is a polygon whose set of vertices is included in the union of the set of vertices of $S$ and $T$. On the contrary, the set of edges of the convex hull of $S$ and $T$ is not included in the union of their set of edges. Indeed, two new "bridging edges," $e_{1}$ and $e_{2}$, appear as illustrated in Fig. 2.a. Actually, efficient algorithms for merging convex hulls are based on finding these two edges [7]. Consider the two lines $l_{1}$ and $l_{2}$ that support $e_{1}$ and $e_{2}$. They divide the image into three regions, namely the region $\mathcal{R}_{S, T}$ on the right with respect to the pair $(S, T)$, the region $\mathcal{L}_{S, T}$ on the left with respect to the same pair and the region $\mathcal{I}_{S, T}$ comprised between the lines (Fig. 2.b). The shape of the latter varies according to the location of their crossing point with respect to the image. The location of a region $U$ with respect to the ordered couple of regions $(S, T)$ of the image is encoded in the chirotope using a rule derived from the case of planar arrangements of points

$$
\chi(S, T, U)= \begin{cases}+ & \text { if } U \in \mathcal{L}_{S, T}, \\ 0 & \text { if } U \in \mathcal{I}_{S, T}, \\ - & \text { if } U \in \mathcal{R}_{S, T} .\end{cases}
$$




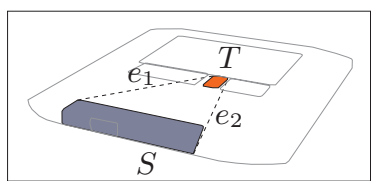

(a)

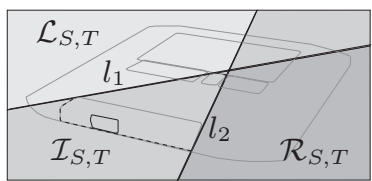

(b)

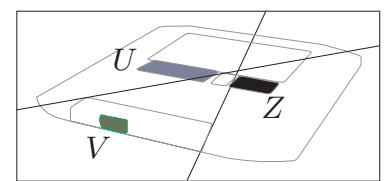

(c)

Fig. 2. Steps of encoding of the combinatorial properties of a view of an object into an oriented matroid

It has been implicitly assumed that $U$ is completely contained into either $\mathcal{R}_{S, T} \mathcal{L}_{S, T}$ or $\mathcal{I}_{S, T}$ but in general it can belong to more that one of them. In this case, since the ratio of areas is an affine invariant, introducing an approximation, we can choose the sign based on which region contains the largest portion of the area of $U$. For instance, if regions $U, V$ and $Z$ are located as in Fig. 2.c we have that $\chi(S, T, U)=+, \chi(S, T, V)=0$ and $\chi(S, T, Z)=-$.

\subsection{Invariance of Oriented Matroids}

Consider a 3D point configuration and one of its views. The combinatorial structure of the $3 \mathrm{D}$ point configuration and that of its $2 \mathrm{D}$ perspective projection are related in the following way: if $x_{0}$ represents in homogeneous coordinates the center of the camera, $p_{0}$, we have that

$$
\operatorname{sign}\left[\bar{x}_{i}, \bar{x}_{j}, \bar{x}_{k}\right]=\operatorname{sign}\left[x_{i}, x_{j}, x_{k}, x_{0}\right]
$$

where $x_{i}, x_{j}$ and $x_{k}$ are the homogeneous coordinates of the $3 \mathrm{D}$ points $p_{i}, p_{j}$ and $p_{k}$, and $\bar{x}_{i}, \bar{x}_{j}$ and $\bar{x}_{k}$ are those of the corresponding points in the view, $\bar{p}_{i}, \bar{p}_{j}$ and $\bar{p}_{k}$. Equation (1) can be regarded as a projection equation for chirotope. It is easy to see that, whereas the matrix that represents in homogeneous coordinates the vertices of a projected set of points is coordinate-dependent, an oriented matroid is a coordinate-free representation. Moreover, the representation of object views based on oriented matroid is a topological invariant, that is, an invariant under homeomorphisms. Roughly speaking, this means that the oriented matroid that represents the arrangement of points of a view of an object does not change when the points undergo a continuous transformation that does not change any orientation of the chirotope. This property makes this representation robust to discretization errors of the image as well as to small changes of the point of view that does not change any orientation of the chirotope. Since projective transformations can be regarded as special homeomorphisms, and we can assert that the representation of the projected set of points based on oriented matroids is projective invariant. However, since affine and Euclidean transformations are special projective transformations, the oriented matroid of the projected set of points of a view of an object does not change under rotations, translations, and affine transformations of the planar arrangement of points themselves. These considerations can be extended to the case in which 

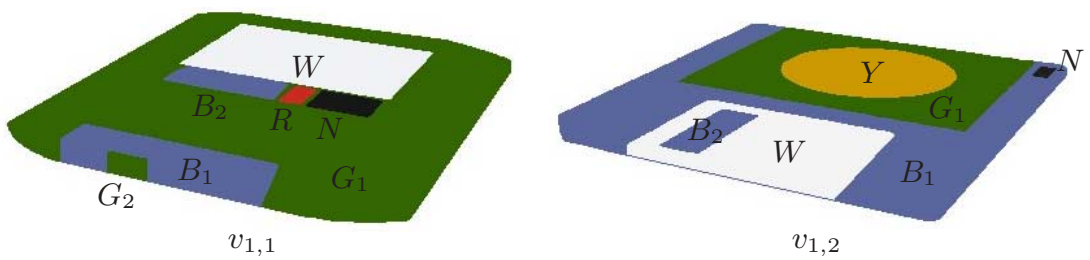

Fig. 3. Two views, of two objects whose combinatorial properties are indexed in Table 4

oriented matroids represent arrangements of planar regions. Therefore, the hypothesis generation method presented in this paper is inherently insensitive to projective, affine and Euclidean transformations of the views.

\section{Indexing Views of 3D Objects}

This process of indexing a database of views of a set of objects starts with some preliminary choices, namely the number of colors in which the hue is quantized and the number of regions having the same color that will be taken into account. These choices, of course, depend on the properties of the images of the database. Then, the views are segmented according tho these choices the set of cocircuits for each view is computed. Then, the sign combinations of the set of cocircuits of the views of the database are merged together and used for indexing a unique table whose entries are spatial combination of features of regions and the records contains the views that contains that combination.

Example 2. In Fig. 3 two views, $v_{1,1}$ and $v_{1,2}$, of two objects are represented, in which a color quantization with 6 colors white $(W)$, red $(R)$, yellow $(Y)$, green $(G)$, blue $(B)$ and black $(N)$ has been applied and up to two regions with the same color are taken into account. Let $(W, R, Y, G, B, N)$ be the ordered tuple of colors considered. The index of the combinatorial properties of these two views is reported in Table 4, in which an asterisk in the first column indicates that the regions are not completely overlapped. On the contrary, a sign + in the same column, in correspondence with a certain ordered couple of regions $(S, T)$, indicates that $S$ completely contains $T$, whereas a - denotes that $S$ is contained in $T$. If they are completely overlapped the corresponding element of the set of cocircuits cannot be computed. An asterisk in the column of the feature $U$ denotes the absence of a region with this feature in the corresponding view of the database. The description of the case of partial overlapping between regions is herein omitted due to space limitations.

\subsection{Hypothesis Generation for Object Recognition}

Given a database of views of a set of 3D objects and a view $v_{i}$ of one of them, not necessarily contained in the database, its set of cocircuits is computed. Each 
Table 4. Index of the combinatorial properties of the two views $v_{1,1}$ and $v_{1,2}$ of the two objects represented in Fig. 3

\begin{tabular}{|c|c|c|c|c|c|c|c|c|c|c|}
\hline & Overlapping & $W$ & $R$ & $Y$ & $G_{1}$ & $G_{2}$ & $B_{1}$ & $B_{2}$ & $N$ & Views \\
\hline$W R$ & $*$ & 0 & 0 & $*$ & 0 & 0 & 0 & - & + & $v_{1,1}$ \\
\hline$W Y$ & $*$ & 0 & $*$ & 0 & 0 & $*$ & 0 & 0 & - & $v_{1,2}$ \\
\hline$W G_{1}$ & - & 0 & $*$ & $*$ & 0 & $*$ & $*$ & $*$ & $*$ & $v_{1,1}$ \\
\hline$W G_{1}$ & $*$ & 0 & $*$ & 0 & 0 & $*$ & 0 & 0 & 0 & $v_{1,2}$ \\
\hline$W G_{2}$ & $*$ & 0 & 0 & $*$ & 0 & 0 & + & 0 & 0 & $v_{1,1}$ \\
\hline$W B_{1}$ & $*$ & 0 & 0 & $*$ & 0 & 0 & 0 & 0 & 0 & $v_{1,1}$ \\
\hline$W B_{1}$ & - & 0 & $*$ & $*$ & $*$ & $*$ & 0 & $*$ & $*$ & $v_{1,2}$ \\
\hline$W B_{2}$ & $*$ & 0 & 0 & $*$ & + & + & + & 0 & + & $v_{1,1}$ \\
\hline$W B_{2}$ & + & 0 & $*$ & $*$ & $*$ & $*$ & $*$ & 0 & $*$ & $v_{1,2}$ \\
\hline$W N$ & $*$ & 0 & 0 & $*$ & - & - & - & - & 0 & $v_{1,1}$ \\
\hline$W N$ & $*$ & 0 & $*$ & + & + & $*$ & 0 & 0 & 0 & $v_{1,2}$ \\
\hline$R Y$ & $*$ & & & & & & & & & \\
\hline$R G_{1}$ & - & $*$ & 0 & $*$ & 0 & $*$ & $*$ & $*$ & $*$ & $v_{1,1}$ \\
\hline$\cdots$ & $\cdots$ & $\cdots$ & $\cdots$ & $\cdots$ & $\cdots$ & $\cdots$ & $\cdots$ & $\cdots$ & $\cdots$ & $\cdots$ \\
\hline
\end{tabular}

element of the set of cocircuits is used to access the table that constitutes the index of the database. For each view $j$ of the object $k, v_{j, k}$, found at that address of the table, the elements $(i, k)$ of an image-object views association matrix are increased of 1 . The final result of the indexing is therefore an association matrix in which the value of the element $(i, k)$ indicates the strength of the hypothesis of associating the image $v_{i}$ with the object $k$ of the database. In other words, the view $v_{i}$ will be associated with the object that has the maximum number of correspondences with $v_{i}$ in terms of cocircuits. It is easy to see that this method for hypothesis generation, that can be regarded as a qualitative version of the geometric hashing technique [8], is also robust to partial occlusions of the objects. Indeed, if a region of a view is occluded, the set of cocircuits can still be computed and the number of correspondences with the views of the database can still be calculated. In this case, obviously, the selectivity of the method decreases.

\section{Experimental Results}

To validate our method, four 3D objects composed by colored woody pieces (Fig. 4) have been created. Then, sixteen views of each of them with angular separation of 22.5 degrees have been taken. These images have been segmented using the segmentation method described in [9]. Then, the index of the learning set of eight views per object taken at the angles $0,45,90,135,180,225,270$ and 315 have been created. In the recognition process, the set of cocircuits of each image of the test set composed by the eight views not used in the learning process, that is, the views taken at angles: $22.5,67.5,115.5,157.5,202.5,247.5$, 292.5 and 337.5 degrees, have been calculated. In this experiment, that should 


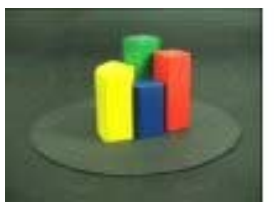

Object 1

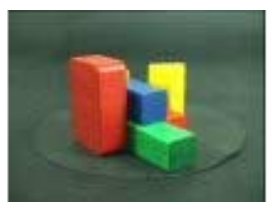

Object 2

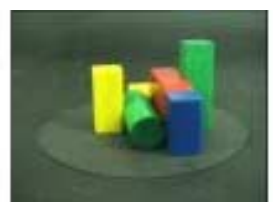

Object 3

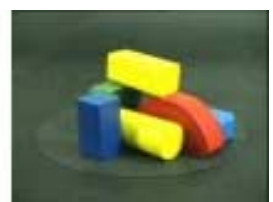

Object 4

Fig. 4. Objects used for the experiments

be regarded as a proof of concept, since the 3D objects employed were not very complex and the images easy to be segmented, all the 32 test views were properly classified.

\section{Conclusions}

In this paper a new method for indexing views of a set of 3D objects has been presented. It is based on oriented matroids, a combinatorial data structure that captures the local and global topology of the regions of the views. This representation is invariant to projective, affine and Euclidean transformation of the views as well as, intrinsically robust to discretization errors of the image and insensitive to small displacements of the point of view. The experimental results obtained applying this indexing technique to the hypothesis generation in $3 \mathrm{D}$ object recognition processes from single views encourage to apply this new method to more complex objects.

\section{References}

[1] Serratosa, F., Alquézar, R., Sanfeliu, A.: Function-described for modeling objects represented by attributed graphs. Pattern Recognition 36 (2003) 781-798 1012

[2] Björner, A., Vergnas, M. L., Sturmfels, B., White, N., Ziegler, G. M.: Oriented Matroids. Volume 43 of Encyclopedia of Mathematics and its Applications. Cambridge University Press (1993) 1013

[3] Bokowski, J., Sturmfels, B.: Computational Synthetic Geometry. Volume 1355 of Lecture Notes in Mathematics. Springer-Verlag (1989) 1013

[4] Richter-Gebert, J., Ziegler, G. M.: Oriented matroids. In Goodman, J. E., O'Rourke, J., eds.: Handbook of Discrete and Computational Geometry. CRC Press (1997) 111-132 1013, 1014

[5] Carlsson, S.: Combinatorial geometry for shape representation and indexing. In: Proceedings of the International Workshop on Object Representation for Computer Vision. (1996) 1013

[6] O' Rourke, J.: Computational Geometry in C. Cambridge University Press (1999) 1015

[7] Toussaint, G.T.: Solving geometric problems with the rotating calipers. In: Proceedings of IEEE MELECON'83, Athens, Greece (1983) 1015

[8] Lamdan, Y., Schwartz, J. T., Wolfson, H. J.: Affine invariant model-based object recognition. IEEE Transactions on Robotics and Automation 6 (1990) 1018 
[9] Comaniciu, D., Meer, P.: Mean shift: A robust approach toward feature space analysis. IEEE Trans. Pattern Anal. Machine Intell. 24 (2002) 603-619 1018 